\title{
Similarities and differences for light-induced surface plasmons in one- and two-dimensional symmetrical metallic nanostructures
}

\author{
C. K. Chang, D. Z. Lin, C. S. Yeh, and C. K. Lee \\ Institute of Applied Mechanics, National Taiwan University, Taipei, Taiwan \\ Y. C. Chang, M. W. Lin, J. T. Yeh, and J. M. Liu \\ Industrial Technology Research Institute, Material and Chemical Research Labs, Hsinchu, Taiwan \\ Received March 21, 2006; revised May 15, 2006; accepted May 15, 2006; \\ posted May 17, 2006 (Doc. ID 69136); published July 10, 2006
}

\begin{abstract}
Two types of double-sided nanostructure, one possessing a slit aperture with parallel grooves and the other possessing a circular aperture with concentric grooves, were fabricated to examine the similarities and differences of their diffraction behavior in one-dimensional (1-D) and two-dimensional (2-D) nanostructures. Based on the projection-slice theory, we conjecture that the surface plasmons in these two different nanoscale grooves possess similar modes. A localized surface plasmon (LSP) was used to examine the transmission characteristics induced by the apertures. The transmission characteristics of the slitted nanostructure and the circular nanostructure aperture were then measured. We coupled the transmission spectra measured from these two apertures with a 1-D parallel groove transmission curve simulated by a 1-D rigorous coupled wave analysis. Measured spectra results show reasonable agreement with the simulated data. We propose that the apparent blueshift observed in the peak frequency of a 2-D nanostructure is due to the difference in the shape of the aperture and the spot transmission characteristics of 1-D and 2-D systems as induced by a LSP. ( 2006 Optical Society of America

OCIS codes: $230.1950,240.6680$.
\end{abstract}

Surface plasmons (SP) require the coupling of light to a metal surface and are characterized by a strong interaction with nanoscale features. According to Bethe's Law, ${ }^{1}$ the light intensity transmitted through a subwavelength aperture is limited. In 1998, Ebbesen et $a l .{ }^{2}$ reported an extraordinary transmission phenomenon: when light is shined on a hole array, most of the incident light beam will be transmitted through the hole array to the other side, even if the hole array is optically thick. In other studies, research has been done on the corrugation around the subwavelength aperture to excite the SP to allow more photons to pass through the aperture. ${ }^{3-9} \mathrm{~A}$ small high-intensity spot that maintains its subwavelength size for up to $1 \mu \mathrm{m}$ was found in this configuration. This finding has triggered much research on this topic because of its vast potential for various applications. ${ }^{10}$ Thus, it would seem that this kind of plasmonic device is a powerful and useful tool that has much potential for a variety of applications.

Equation (1) shows how the SP is generated by the nanostructure:

$$
\vec{K}_{\text {sp }}=\vec{K}_{\text {incident }} \pm n \vec{K}_{\text {nanostructure }}
$$

When the light beam impinges on a periodic nanostructure, the nanostructure will provide additional photon momentum. If the nanostructure provides a large enough momentum with incident light, the incident light wave will become an evanescent wave that propagates along the surface. This is unlike the situation for dielectrics, as no SP will appear in the interface between two dielectric materials due to their lack of many free electrons. When a lightpassage channel exists to link the two surfaces pos- sessing nanostructured features, i.e., the incident from the upper side and the exit at the lower side, the light energy gets carried forward through the channel by the electric charge oscillations. If the exit side also has a periodic nanostructure, the evanescent wave (SP wave) will be converted into a free-space light beam by the added photon momentum induced by the nanostructure. Equation (2) shows the mechanism $^{11}$ :

$$
I=f_{\text {in }} T f_{\text {out }},
$$

where $I$ is the re-emitted light intensity; $f_{\text {in }}$ and $f_{\text {out }}$ are the transfer functions of the incident and the exit side, respectively; and $T$ is the transfer function of the channel. More specifically, the intensity of the reemitted light beam from the aperture will be governed by three independent terms. The variables $f_{\text {in }}$ and $f_{\text {out }}$ depend on the period, depth, and duty cycle of the periodic structure and $T$ depends on the geometry of the channel as well as the thickness of the metal film. Although the mechanism of extraordinary transmission in 1-D and 2-D systems has been discussed previously, ${ }^{12}$ no linkage between the SP modes in 1-D and 2-D systems has been discussed in detail. As it is relatively easy to simulate the performance of a 1-D nanostructure, this certainly represents an intriguing perspective from which to examine the possibility of understanding the performance of a 2-D nanostructure by using a 1-D nanostructure simulation. However, it is also important to know the limitations of such an approach. Both perspectives have led to the following studies in our work and signify the underlying goal of this Letter.

Experimental results were used to explore the similarities in the SP modes of 1-D and 2-D nano- 


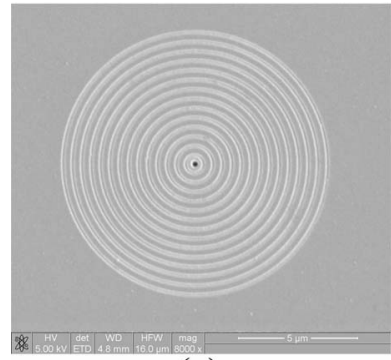

(a)

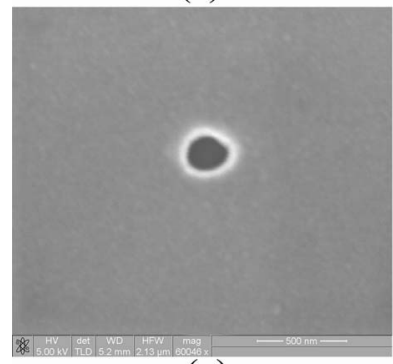

(c)

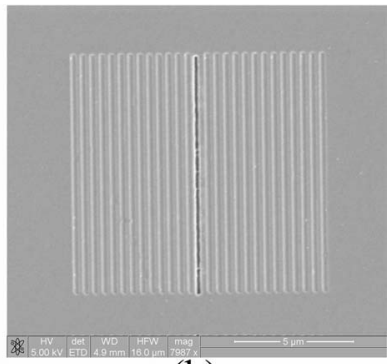

(b)

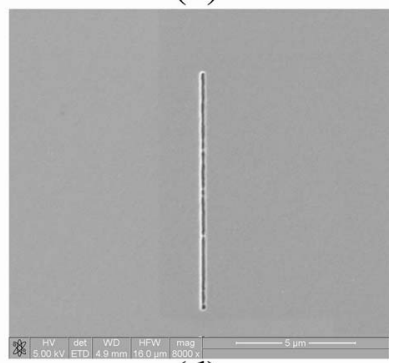

(d)
Fig. 1. Fabricating nanostructures by using FIB: (a) 2-D structure, (b) 1-D structure, (c) single $200 \mathrm{~nm}$ diameter hole, (d) single $100 \mathrm{~nm}$ wide and $10 \mu \mathrm{m}$ long slit.

structures that possess the same geometrical conditions such as pitch, grating depth, and grating profile. We defined a 1-D nanostructure as having a slit surrounded by parallel grooves and a 2-D structure as having a hole surrounded by circular grooves. All samples were fabricated by using the following steps. A thin Cr film was sputtered onto a glass wafer to provide a focusing target for the focused ion beam (FIB) system (FEI Nova 200). Once focused, the FIB was used to etch a periodic structure directly onto the glass substrate. The designed groove depth, period, and duty cycle were $50 \mathrm{~nm}, 450 \mathrm{~nm}$, and $30 \%$, respectively, for the 1-D and 2-D grating systems. Once the substrate was etched, the $\mathrm{Cr}$ film was removed to avoid any potential SP property changes induced by the film. Then, approximately $250 \mathrm{~nm} \mathrm{Ag}$ film was deposited. A FIB was then used to drill both a slit in the center of the 1-D structure and a hole in the center of the 2-D structure. The slit was $100 \mathrm{~nm}$ wide by $10 \mu \mathrm{m}$ long, and the diameter of the hole was $200 \mathrm{~nm}$. Figures 1(a) and 1(b) show micrographs of these two structures.

We then captured the normal incidence optical spectra shown in Fig. 2(a), using a confocal microscope equipped with a spectrometer. Illumination was provided by a halogen lamp and the signal detected by a nitrogen-cooled CCD. Figure 2(a) shows the experimental results obtained when the light beam was incident along the normal direction.

It is clear from Fig. 2(a) that both nanostructure spectra data have the same number of peaks, even though an apparent blueshift phenomenon was found in the 2-D structure and not in the 1-D structure. We propose that this shift can be attributed to the localized surface plasmon (LSP). When the light beam impinges on a small metal defect, the induced electric dipole (i.e., the LSP) radiates an electromagnetic wave. The LSP ${ }^{13,14}$ modes are thus determined by defect geometry. It is clear from Eq. (2) that the trans- mitted light efficiency is determined by three independent terms. Even though both the 1-D and 2-D grooves possess basically the same geometry, the aperture geometry appears to influence the optical transmission characteristics. To understand this effect, a single hole and a single slit [Figs. 1(c) and 1(d)] of the same dimensions were fabricated. The transmission spectra of the single hole and the slit are different, as can be seen in Fig. 2(b). It is clear from Fig. 2(b) that the dispersion relationship for a single slit favors a longer wavelength. It will be shown below that this phenomenon is the primary contribution of the apparent blueshift difference in the two spectra [see Figs. 2(a) and 2(b)].

Attempting to understand the above-mentioned physical phenomenon by using Eq. (2), we examined the effect of a 1-D periodic structure on the incident side by using a rigorous coupled wave analysis (RCWA) method. ${ }^{15}$ We calculated the reflection and transmission efficiency on interfaces 1 and 2, respectively, as shown in Fig. 3. Figure 3 details the relationship between the reflectivity and the incident light beam wavelength, which shows two reflectivity minima. These two reflectivity minima represent the two main modes associated with the interaction of the light beam and the metallic nanostructure. The halogen lamp light source was randomly polarized. For this reason, we took the average of TM (polarization perpendicular to the groove) and TE (polarization parallel to the groove) of the SP mode simulation results. As the atomic force microscope measurements along the transverse direction are known to have a $3 \%$ inaccuracy and the nominal groove depth, period, and duty cycle were set to be $50 \mathrm{~nm}, 450 \mathrm{~nm}$,

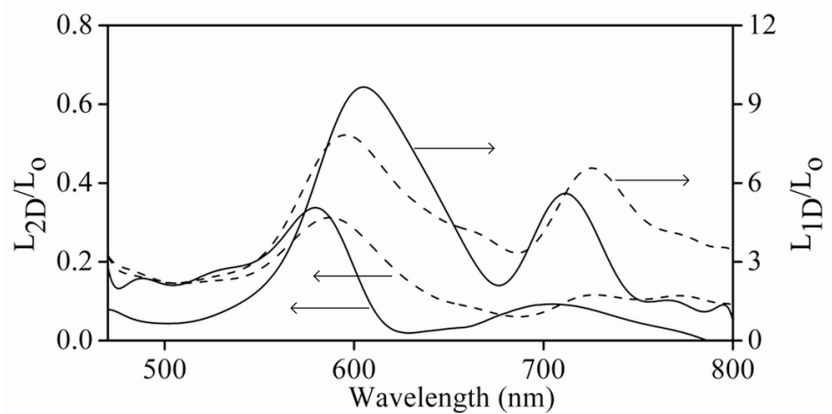

(a)

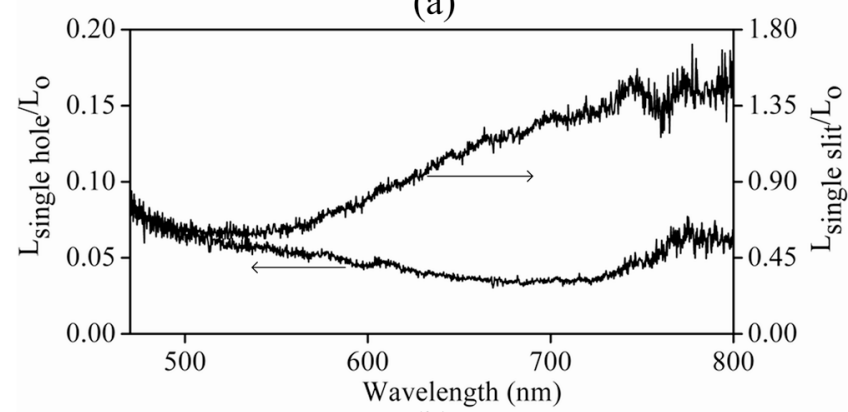

(b)

Fig. 2. Transmission spectra and $L_{o}$ intensity of light source: (a) 1-D and 2-D structure systems (the two dashed curves are the results of simulation and experimental data) and (b) single hole and single slit (the direction of each arrow identifies the corresponding axis of each curve). 


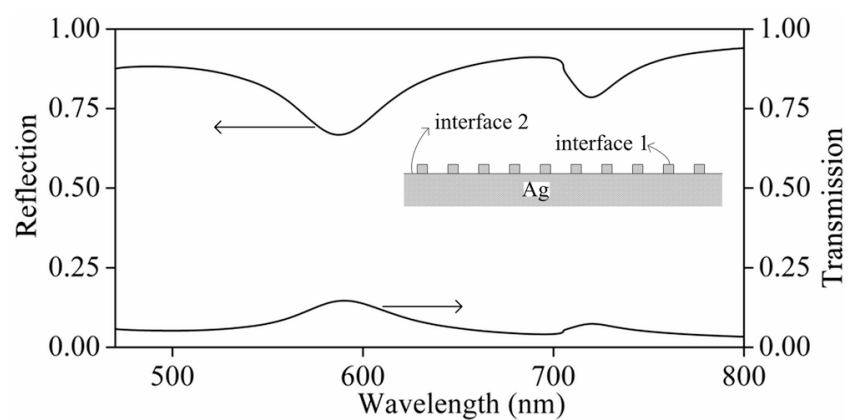

Fig. 3. Reflection and transmission spectra at the incident side.

and $30 \%$ respectively, we simulated a period and a duty cycle of the grating from 436.5 to $463.5 \mathrm{~nm}$ and from $29.1 \%$ to $30.9 \%$, respectively. By matching these results to the experimental results, we determined and adopted $50 \mathrm{~nm}, 460 \mathrm{~nm}$, and $29.3 \%$ as the simulated groove depth, period, and duty cycle, respectively. Note that the transmitted energy calculated is the energy arriving at the light-passage channel, i.e., energy transmitted through interface 2 and right before region $\alpha$ (Fig. 3). For comparison, the reflective energy on interface 1 and the transmitted energy on interface 2 are shown in Fig. 3. We adopted published refraction indices at different wavelengths from a handbook $^{16}$ and interpolated the values not listed. The refraction index of the incident side was set at 1.53. The two transmission maxima corresponded to the SP modes transmitted through the light-passage channel in the metal medium, as shown by the spectrometer measurement data. The two dashed curves in Fig. 2(a) are the product of the measured data shown in Fig. 2(b) and the transmission data shown in Fig. 3. Despite some small peak frequency discrepancies found between the theoretical prediction and the experimental data in Fig. 2(a), the SP mode shift induced by the LSP can still be clearly observed. From our findings, we propose that the transmission characteristics of a 2-D and a 1-D nanostructure are similar, except for the observation of an apparent blueshift that we can explain with our experimental results [see Fig. 2(b)]. By analysis of previous studies in the literature, we can find a basis for our proposition. One previous study showed that free-space propagating diffraction angles can be examined by using the far-field optical diffraction theory. ${ }^{17}$ As we know, a Fourier transform can be used to examine the far-field diffraction behavior of a grating. Along this same line, we know that the projection-slice theory ${ }^{18}$ states that a 1-D Fourier transform $P_{\theta}\left(s^{\prime}\right)$ of a projection $p_{\theta}\left(x^{\prime}\right)$ through $f(x, y)$ will be identical to a 2-D transform $F\left(s_{x}, s_{y}\right)$ of $f(x, y)$ evaluated along a slice through the origin. As the two kinds of surface nanostructure discussed in this Letter have the same spatial frequency, which adds photon momentum to couple the incident light into the SP, the projectionslice theory essentially implies that the light beam will experience similar coupling responses for these two types of structure. More specifically, we can use a 1 -D algorithm to approximate a $2-\mathrm{D}$ experimental re- sult if the prediction is related to finding the number of coupling modes, etc.

In summary, a 1-D simulation based on a RCWA algorithm predicts two minima, which translates to two coupling modes. The two modes were simulated to have their resonances at 580 and $710 \mathrm{~nm}$, as shown in Fig. 3, which agrees well with the experimental results [see Fig. 2(a)]. Additionally, the projection-slice theory essentially provides us with a path to extend the behavior of the resonant modes from a 1-D to a 2-D system, i.e., the existence of two resonance modes at 580 and $710 \mathrm{~nm}$. However, the data show that an apparent blueshift behavior will be present and will induce a peak shift in the 2-D system. From this, we can set the SP mode in a circular groove to a 1-D RCWA algorithm, which can save much computation time and effort compared with the costly calculations needed for a 2 -D periodic surface nanostructure.

This work is partially supported by the Materials Research Laboratory of the Industrial Technology Research Institute and by the National Science Council under project 94-2212-E-002-005. C. K. Lee's e-mail address is cklee@mems.iam.ntu.edu.tw.

\section{References}

1. H. A. Bethe, Phys. Rev. 66, 163 (1944).

2. T. W. Ebbesen, H. J. Lezec, H. F. Ghaemi, T. Thio, and P. A. Wolff, Nature 391, 667 (1998).

3. H. J. Lezec, A. Degiron, E. Devaux, R. A. Linke, L. Martin-Moreno, F. J. Garcia-Vidal, and T. W. Ebbesen, Science 297, 820 (2002).

4. L. Martin-Moreno, F. J. Garcia-Vidal, H. J. Lezec, A. Degiron, and T. W. Ebbesen, Phys. Rev. Lett. 90, 167401 (2003).

5. T. Thio, H. J. Lezec, T. W. Ebbesen, K. M. Pellerin, G. D. Lewen, A. Nahata, and R. A. Linke, Nanotechnology 13, 429 (2002).

6. T. Thio, K. M. Pellerin, and R. A. Linke, Opt. Lett. 26, 1972 (2001).

7. T. Ishi, J. Fujikata, and K. Ohashi, Jpn. J. Appl. Phys. 44, 170 (2005).

8. W. L. Barnes, A. Dereux, and T. W. Ebbesen, Nature 424, 824 (2003).

9. T. Thio, H. J. Lezec, and T. W. Ebbesen, Physica B 279, $90(2000)$.

10. C. K. Lee, C. L. Lin, D. Z. Lin, T. D. Cheng, C. K. Chang, L. B. Yu, and C. S. Yeh, Mater. Sci. Forum 505-507, 1 (2006).

11. A. Degiron and T. W. Ebbesen, Opt. Express 12, 3694 (2004)

12. F. J. Garcia-Vidal, H. J. Lezec, T. W. Ebbesen, and L. Martin-Moreno, Phys. Rev. Lett. 90, 213901 (2003).

13. A. Degiron, H. J. Lezec, N. Yamamoto, and T. W. Ebbesen, Opt. Commun. 239, 61 (2004).

14. A. Degiron and T. W. Ebbesen, J. Opt. A, Pure Appl. Opt. 7, S90 (2005).

15. M. G. Moharam and T. K. Gaylord, J. Opt. Soc. Am. A 3, 1780 (1986).

16. E. D. Palik, Handbook of Optical Constants of Solids (Academic, 1985).

17. L. B. Yu, D. Z. Lin, Y. C. Chen, Y. C. Chang, K. T. Huang, J. W. Liaw, J. T. Yeh, J. M. Liu, C. S. Yeh, and C. K. Lee, Phys. Rev. B 71, 041405 (2005).

18. R. N. Bracewell, Fourier Transform and Its Applications (McGraw-Hill, 2000). 